\title{
On the Asymptotic Capacity of Dual-Aperture FSO Systems with Generalized Pointing Error Model
}

\author{
Hessa AlQuwaiee, Student Member, IEEE, Hong-Chuan Yang, Senior Member, IEEE, and Mohamed-Slim \\ Alouini, Fellow, IEEE
}

\begin{abstract}
Free-space optical (FSO) communication systems are negatively affected by two physical phenomenon, namely, scintillation due to atmospheric turbulence and pointing errors. To quantify the effect of these two factors on FSO system performance, we need an effective mathematical model for them. In this paper, we propose and study a generalized pointing error model based on the Beckmann distribution. We then derive a generic expression of the asymptotic capacity of FSO systems under the joint impact of turbulence and generalized pointing error impairments. Finally, the asymptotic channel capacity formula are extended to quantify the FSO systems performance with selection and switched-and-stay diversity.
\end{abstract}

Index Terms-Free-space optical, log-normal fading, GammaGamma fading, Ergodic capacity, asymptotic, high SNR, pointing errors, Beckmann distribution, correlated branches.

\section{INTRODUCTION}

One of the potential solutions to the spectrum scarcity problem is optical wireless communications (OWC), which utilizes the unlicensed optical spectrum. Long-range outdoor OWC are usually referred to as free-space optical (FSO) communications in the literature. Unlike radio frequency (RF) communications, FSO is immune to interference and multipath fading. Also, the deployment of FSO systems is flexible and much faster than optical fibers. These attractive features make FSO applicable for broadband wireless transmission such as optical fiber backup, metropolitan area network, and last mile access.

Although FSO communications is a promising technology, it still faces challenges that prompted intensive research in the last decade. One of the primary concern is that weather conditions such as fog and snow can cause some attenuation in the intensity of the laser beam. Moreover, FSO is prone to atmospheric turbulence in which different intensity air layers formed locally by temperature differences vary the refractive index leading to scintillation of the laser beam [1]. In addition, the misalignment between the transmitter and the receiver leads to pointing error and additional performance degradation. The misalignment originates from either mechanical error in the tracking system or mechanical vibrations in the system due to winds or/and building sway [2]. More specifically, pointing error results from the displacement of the laser beam along vertical (elevation) and horizontal (azimuth) directions that are typically assumed to be independent Gaussian random variables (RVs). A pointing error has two main components: the boresight and the jitter. The boresight is caused by thermal expansion of the laser beam and defined as the fixed displacement between the beam footprint center and the center of detection plane. On the other hand, the jitter is the random offset of the beam center at the detector plane, typically caused by building sway, weak earthquakes, and dynamic wind loads [3].

As widely adopted in RF, spatial diversity, in which multiple transmitter and/or multiple receivers are employed, is usually utilized to suppress the effect of channel fading. Similarly, this technique can efficiently overcome scintillation according to some reported work in the literature [4], [5]. However, when considering diversity links and due to system design, it is crucial to take into account the correlation of the underlying channels since the spacing between beams or apertures can not always ensure uncorrelated signals [6]. Furthermore, identifying the statistical model of the received irradiance that counts for both scintillation and pointing error facilities the performance analysis of FSO systems especially in diversity links. To illustrate, the scintillation effect is widely modeled as Log-Normal (LN) for weak turbulence conditions and GammaGamma (ГГ) for strong turbulence [7]-[13]. In terms of the pointing error, it was first modeled in [14] by a Rayleigh distribution, in [15] by a Hoyt distribution, in [3] by a Rician distribution and more generally by a Beckmann distribution ${ }^{1}$ in the conference version of this paper [17]. Moreover, combined statistics of turbulence and pointing errors were too investigated for Gamma-Gamma $\backslash$ Rayleigh channel model in [18], for double generalized Gamma $\backslash$ Rayleigh channel in [19], for Log-Normal $\backslash$ Rician in [3], and for Málaga $(\mathcal{M}) \backslash$ Rayleigh channel model in [20]. Finding the combined effect of pointing errors and turbulence becomes a harder task when considering general models of each (i.e. considering Beckmann model for the pointing error effect) and even more complicated for spatial diversity systems [21]-[24]. Hence, obtaining closed-form expression of any of the performance measures might not be feasible especially in the case of the irradiance being a mixture of two independent processes. This has raised the interest to investigate the asymptotic limit at high signal-to-noise ratio (SNR). In this work, our interest is one of the fundamental information-theoretic measures namely channel capacity. At high SNR regime, it can be easily derived by utilizing the $n$th moment of the effective receive SNR. Interestingly, this approach can be utilized as well to find the asymptotic capacity of diversity links.

Several research work have been reported in the literature

\footnotetext{
${ }^{1}$ The Beckmann distribution [16] is a four-parameter distribution corresponding to the envelope of two independent Gaussian random variables, each with their own mean and variance. It is different than the Log-Normal Rican distribution which can be also called as Beckmann distribution [8].
} 
Table I: Summary of research work on ergodic capacity.

\begin{tabular}{c|c|c|c}
\hline \hline Detection Technique & Channel Model & Turbulence Only & Turbulence and Pointing Error \\
\hline \multirow{4}{*}{ IM/DD } & $\Gamma \Gamma$ & {$[11],[25],[26]$} & {$[27],[18]$} \\
\cline { 2 - 4 } & LN & {$[11],[25]$} & $*$ \\
\cline { 2 - 4 } & Other & {$[28][29]$} & {$[19]$} \\
\hline \multirow{2}{*}{$\begin{array}{c}\text { Heterodyne } \\
\text { Detection }\end{array}$} & $\Gamma \Gamma$ & $*$ & {$[30],[18]$} \\
\cline { 2 - 4 } & LN & $*$ & $*$ \\
\cline { 2 - 4 } & Other & $*$ & {$[19]$} \\
\hline
\end{tabular}

aiming to find closed-form expressions of the ergodic capacity of FSO systems as summarized in Table. I. It is noticed that the studies focusing on the joint effect of turbulence and pointing errors assume zero boresight and same jitter variance for horizontal and vertical misalignment. In our work, we try to generalize the pointing error model. Thus, our contribution in this paper is twofold. First, we derive closed-form asymptotic results of the ergodic capacity of single link FSO system under weak and strong turbulence conditions and under the impact of generalized pointing error impairments. Our result is general for any turbulence channel model and for any scenario of the pointing error. Second, we extend our result to be utilized in the derivation of the channel capacity of FSO systems with diversity reception. In particular, we obtain closed-form asymptotic expressions of the capacity of dualbranch correlated FSO channels considering general model of the pointing error.

The remainder of the paper is organized as follows. In Section II, system and channel model of turbulence and pointing error are described. In Section III, analytical expressions of the channel capacity for single and dual-aperture FSO system are obtained. Section IV highlights some numerical examples of the results and finally concluding remarks are given in Section V. $\Phi$

\section{Channel And System Model}

\section{A. System Model}

In this work, we consider single-input single-output (SISO) and dual-branch single-input multiple-output (SIMO) configurations of the FSO system with two types of detection techniques, heterodyne and intensity modulation/direct detection (IM/DD). Data transmission is affected by path loss, atmospheric turbulence conditions, pointing errors, and additive white Gaussian noise $(\mathrm{AWGN})^{2}$. As such, the received vector $\mathbf{y}=\left[\begin{array}{ll}y_{1} & y_{2}\end{array}\right]^{T}$ is given by

$$
\mathbf{y}=\eta \mathbf{I}_{\mathbf{a}} \mathbf{I}_{\mathbf{p}} x+\boldsymbol{\omega},
$$

where $\eta$ is the effective photoelectric conversion ratio, $\mathbf{I}_{\mathbf{a}}=$ $\operatorname{diag}\left(I_{1}, I_{2}\right)$ is a $2 \times 2$ diagonal matrix reflects the turbulenceinduced fading such that $I_{i}$ represents the irradiance received at the $i$ th aperture, $\mathbf{I}_{\mathbf{p}}=\left[\begin{array}{ll}I_{p_{1}} & I_{p_{2}}\end{array}\right]^{T}$, with $(.)^{T}$ is the transpose

\footnotetext{
${ }^{2}$ Most optical wireless systems operate in shot-noise limited regime and in that case the ambient light shot noise component dominants the shot noise generated from signal and the circuit. Thus, the resulting noise of the channel becomes white shot noise and can be distributed as Poisson random variables. By the central limit theorem, as the number of random variables approaches infinity, the cumulative distribution function is approximated by Gaussian distribution.
}

operator, is the $2 \times 1$ pointing error matrix consists of independent random variables where each component represents the misalignment between the center of the beam footprint and the center of the $i$ th aperture, and $x \in\left\{0,2 P_{t}\right\}$ is the onoff keying $(\mathrm{OOK})$ modulated transmitted signal with $P_{t}$ being average transmitted optical power. The vector $\boldsymbol{\omega}=\left[\omega_{1} \omega_{2}\right]^{T}$ is a noise vector of independent components modeled as white and Gaussian distributed RVs. It is important to note that $I_{i}$ 's are not necessarily independent random variables. The spatial matrix $R$ can be of the form

$$
R=\left[\begin{array}{ll}
1 & \rho \\
\rho & 1
\end{array}\right],
$$

where $\rho$ is the correlation between $I_{1}$ and $I_{2}$. The electrical SNR of the $i$ th branch can be defined as

$$
\gamma_{i}=\frac{\left(\eta I_{i} I_{p_{i}}\right)^{r}}{N_{0}},
$$

where $r$ depends on the detection techniques: $r=1$ for heterodyne and $r=2$ for IM/DD. Hence, the average electrical SNR, $\mu$, is then expressed as

$$
\mu=\frac{\eta^{r} \mathbb{E}\left[I_{i}\right]^{r} \mathbb{E}\left[I_{p_{i}}\right]^{r}}{N_{0}},
$$

where $\mathbb{E}[$.$] denotes the expectation operator. Then, the n$th moment of the electrical SNR can be written as

$$
\mathbb{E}\left[\gamma_{i}^{n}\right]=\frac{\mathbb{E}\left[I_{i}^{r n}\right] \mathbb{E}\left[I_{p_{i}}^{r n}\right]}{\mathbb{E}\left[I_{i}\right]^{r n} \mathbb{E}\left[I_{p_{i}}\right]^{r n}} \mu^{n} .
$$

\section{B. Channel Model}

1) Atmospheric Turbulence: The atmospheric turbulence can be classified into two categories: large-scale (diffractive) and small-scale fluctuations (refractive) (i.e. if the turbulence cells are larger than the beam diameter and vise versa). Moreover, the resulting irradiance can be modeled as

$$
I_{i}=I_{x} I_{y},
$$

where $I_{x}$ and $I_{y}$ are statistically independent unit mean RVs representing large-scale and small-scale fluctuations, respectively. For instant, assuming plane wave, turbulence conditions can be categorized into three regimes according to the Rytov variance, $\sigma_{R_{i}}^{2}$ [31]: a weak fluctuations regime $\left(\sigma_{R_{i}}^{2}<0.3\right)$, a moderate-fluctuations regime $\left(0.3 \leq \sigma_{R_{i}}^{2}<5\right)$, and a strong fluctuations regime $\left(\sigma_{R_{i}}^{2} \geq 5\right)$.

For weak turbulence conditions, in which large-scale fluctuations dominate, $I_{i}$ is modeled as [7].

$$
I_{i}=\exp \left(2 X_{i}\right),
$$


where $X_{i} \sim \mathcal{N}\left(\mu_{X_{i}}, \sigma_{X_{i}}^{2}\right)$ is the log-amplitude of the optical intensity such that $\sigma_{X_{i}}^{2} \approx \sigma_{R_{i}}^{2} / 4=0.30545 k^{7 / 6} C_{n_{i}}^{2} z^{11 / 6}$ where $k=2 \pi / \lambda$ is the optical wavenumber with $\lambda$ being the wavelength, $z$ being the transmission distance, and $C_{n_{i}}^{2}$ is the index of refraction structure parameter of atmosphere ${ }^{3}$. The refractive index is considered as one of the most critical parameters to measure the strength of turbulence [7]. It is an altitude dependent parameter defined as

$$
\begin{aligned}
C_{n}^{2}(h) & =0.00594\left(\frac{t}{27}\right)^{2}\left(10^{-5} h\right)^{10} \exp \left[-\frac{h}{1000}\right] \\
& +2.7 \times 10^{-16} \exp \left[-\frac{h}{1500}\right]+A \exp \left[-\frac{h}{100}\right],
\end{aligned}
$$

such that $h$ is the height, $t$ is the wind speed in meters per second and $C_{0}$ is the nominal value of the refractive index at the ground which is estimated to be $1.7 \times 10^{-14}$. Typical $C_{n}^{2}$ values can vary from $10^{-17} \mathrm{~m}^{\frac{2}{3}}$ to $10^{-12} \mathrm{~m}^{\frac{2}{3}}$ indicating weak atmospheric turbulence and strong conditions, respectively [25]. Then the probability density function (PDF) of $I_{i}$ can be given as

$$
f_{I_{i}}(I)=\frac{1}{I \sigma_{I_{i}} \sqrt{2 \pi}} \exp \left\{-\frac{\left(\ln (I)-\mu_{I_{i}}\right)^{2}}{2 \sigma_{I_{i}}^{2}}\right\},
$$

where $\mu_{I_{i}}=2 \mu_{X_{i}}$ and $\sigma_{I_{i}}^{2}=4 \sigma_{X_{i}}^{2}$ are the mean and standard deviation of $I_{i}$. To ensure that the average power is not amplified by fading, the irradiance is normalized (i.e $\mathbb{E}\left[I_{i}\right]=1$ and $\left.\mu_{I_{i}}=-2 \sigma_{X_{i}}^{2}\right)$ [21]. The $n$th moment for a log-normal RV $I_{i}$ can then be calculated as

$$
\mathbb{E}\left[I_{i}^{n}\right]=\exp \left(n \mu_{I_{i}}+\frac{n^{2} \sigma_{I_{i}}^{2}}{2}\right)=\exp \left(2 n \sigma_{X_{i}}^{2}(n-1)\right) .
$$

On the other hand, moderate-to-strong turbulence conditions resulted from combined effect of the large-scale and smallscale fluctuations, leading to $I_{i}$ modeled as $Г \Gamma \mathrm{RV}$ with a PDF given by [8]

$$
f_{I_{i}}(I)=\frac{2\left(\alpha_{i} \beta_{i} I\right)^{\frac{\alpha_{i}+\beta_{i}}{2}}}{\Gamma\left(\alpha_{i}\right) \Gamma\left(\beta_{i}\right) I} K_{\alpha_{i}-\beta_{i}}\left(2 \sqrt{\alpha_{i} \beta_{i} I}\right),
$$

where $\Gamma($.$) is the Gamma function, K_{j}($.$) is the modified$ Bessel function of order $j, \alpha_{i}$ and $\beta_{i}$ are the fading parameters of large-scale and small-scale fluctuations, defined as [8, Eq. 14] in the case of plane wave

$$
\begin{aligned}
\alpha_{i} & =\left[\exp \left(\frac{0.49 \sigma_{R_{i}}^{2}}{\left(1+1.11 \sigma_{R_{i}}^{12 / 5}\right)^{7 / 6}}\right)-1\right]^{-1}, \\
\beta_{i} & =\left[\exp \left(\frac{0.51 \sigma_{R_{i}}^{2}}{\left(1+0.69 \sigma_{R_{i}}^{12 / 5}\right)^{5 / 6}}\right)-1\right]^{-1} .
\end{aligned}
$$

The PDF in Eq. (10) can be rewritten in terms of the Meijer's

\footnotetext{
${ }^{3}$ For plane wave propagation, the Rytov variance is given as $\sigma_{R_{i}}^{2}=$ $1.23 k^{7 / 6} C_{n_{i}}^{2} z^{11 / 6}$ and $\sigma_{X_{i}}^{2}=0.30545 k^{7 / 6} C_{n_{i}}^{2} z^{11 / 6}$. However, for spherical wave propagation, the Rytov variance is equal to $\sigma_{R_{i}}^{2}=0.5 k^{7 / 6}$ $C_{n_{i}}^{2} z^{11 / 6}$ and therefore, $\sigma_{X_{i}}^{2}=0.1250 k^{7 / 6} C_{n_{i}}^{2} z^{11 / 6}$ [7, Eq. (97)].
}

G-function as

$$
f_{I_{i}}(I)=\frac{\left(\alpha_{i} \beta_{i} I\right)^{\frac{\alpha_{i}+\beta_{i}}{2}}}{\Gamma\left(\alpha_{i}\right) \Gamma\left(\beta_{i}\right) I} \mathrm{G}_{0,2}^{2,0}\left[\alpha_{i} \beta_{i} I \mid \begin{array}{c}
\frac{\alpha_{i}-\beta_{i}}{2}, \frac{\beta_{i}-\alpha_{i}}{2}
\end{array}\right],
$$

where $G_{., .,}[$.$] is the Meijer G-function. Next, the n$th moment of $I_{i}$ can be derived by utilizing [32, Eq. 07.34.21.0009.01] as

$$
\mathbb{E}\left[I_{i}^{n}\right]=\frac{\Gamma(\alpha+n) \Gamma(\beta+n)}{(\alpha \beta)^{n} \Gamma(\alpha) \Gamma(\beta)} .
$$

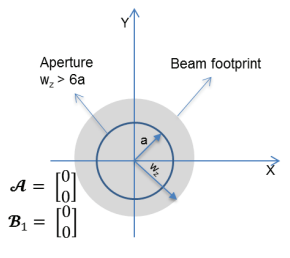

(a) Zero boresight: No misalignment.

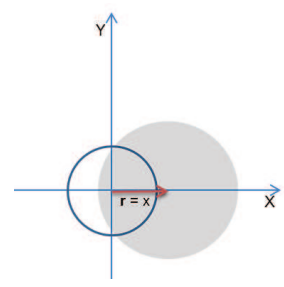

(c) Uni-Directional misalignment: zero boresight [22].

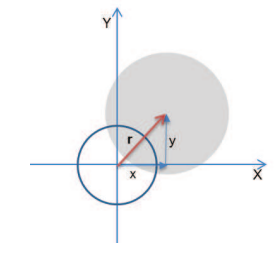

(b) Bi-Directional misalignment: zero boresight and identical jitters [14].

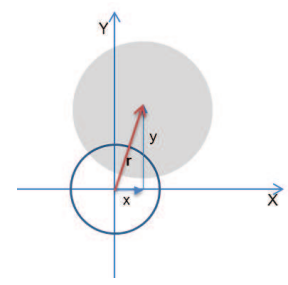

(d) Bi-Directional misalignment: zero boresight and non-identical jitters.

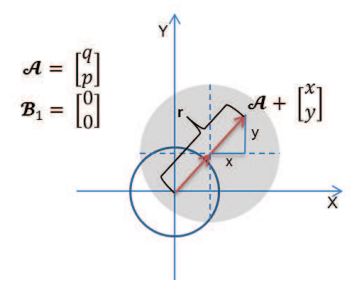

(e) Bi-Directional misalignment: non-zero boresight and identical jitters.

Figure 1: Beam footprint on the detector plane.

2) Pointing Error Generalized Model: Assuming Gaussian beam with initial beamwaist, $w_{0}$, and radius of curvature, $F_{0}$, propagating through atmospheric turbulence of distance $z$, the beam waist at the receiver in long term, $w_{z}$, can be defined [33, Eq. 45, p.238].

For the SISO setup as shown in Fig. 1a, the beam is initially pointing at $\mathcal{A}=[q p]^{t}$ in the detection plane and it is detected by the $i$ th aperture of radius $a$ placed at $\mathcal{B}_{i}=\left[\begin{array}{ll}u_{i} & v_{i}\end{array}\right]^{t}$ (i.e. $i=1$ for the SISO case). Moreover, due to important initialization or other effect, $\mathcal{A}$ may not collocated with $\mathcal{B}_{i}$. Also, the beam may experience random displacements in two directions namely, horizontal, $x$ and vertical, $y$ as the result of building sway. It is commonly assumed that both displacements are modeled as independent Gaussian random variable i.e. $x \sim \mathcal{N}\left(\mu_{x}, \sigma_{x}\right)$ and $y \sim \mathcal{N}\left(\mu_{y}, \sigma_{y}\right)$. Then the distance between the center of the beam footprint and the 
center of the $i$ th aperture can be expressed as

$$
r_{i}=\left\|\mathcal{A}-\mathcal{B}_{i}+\left[\begin{array}{l}
x \\
y
\end{array}\right]\right\|=\left\|\left[\begin{array}{l}
\hat{x} \\
\hat{y}
\end{array}\right]\right\| .
$$

It follows that the attenuation due to geometric spread and pointing errors can be approximated by [14]

$$
I_{p_{i}}\left(r_{i}: z\right) \approx A_{0} \exp \left(-\frac{2 r_{i}^{2}}{w_{z_{e q}}^{2}}\right),
$$

where $r_{i}=\sqrt{\hat{x}^{2}+\hat{y}^{2}}$ such that $\hat{x} \sim \mathcal{N}\left(\hat{\mu}_{x}, \sigma_{x}^{2}\right)$ and $\hat{y} \sim \mathcal{N}\left(\hat{\mu}_{y}, \sigma_{y}^{2}\right), \hat{\mu}_{x}=\mu_{x}+q-u_{i}, \hat{\mu}_{y}=\mu_{y}+p-v_{i}$, and $w_{z_{e q}}^{2}$ is the equivalent beamwidth defined as $w_{z_{e q}}^{2}=w_{z}^{2} \frac{\sqrt{A_{0} \pi}}{2 g \exp \left\{-g^{2}\right\}}$ such that $A_{0}=[\operatorname{erf}(g)]^{2}$ is the maximum fraction of the collected power (i.e. the fraction of power at $r_{i}=0$ ), and $g=\sqrt{\frac{a^{2} \pi}{2 w_{z}^{2}}}$ is the ratio between the aperture radius and the beamwidth. It is important to note that the approximation in (16) is valid when $w_{z}>6 a$ [14]. Moreover, the distribution of $r_{i}$ depends on $\hat{x}=x+q-u_{i}$ and $\hat{y}=y+p-v_{i}$. Since $\hat{x}$ and $\hat{y}$ are independent Gaussian RVs, then $r_{i}$ can be distributed according to the following distributions.

a) Beckmann Distribution: The Beckmann distribution is a versatile model that includes many distributions as special cases. It is a four-parameter distribution modeling the envelope of two independent Gaussian RVs. In our case, if both displacements are nonzero mean Gaussian RVs with different jitters, i.e. $\hat{x} \sim \mathcal{N}\left(\hat{\mu}_{x}, \sigma_{x}^{2}\right)$ and $\hat{y} \sim \mathcal{N}\left(\hat{\mu}_{y}, \sigma_{y}^{2}\right)$, then $r_{i}$ follows the Beckmann distribution [34, Eq. 2.37] with probability density function (PDF) given by

$$
\begin{aligned}
f_{r}(r) & =\frac{r}{2 \pi \sigma_{x} \sigma_{y}} \\
& \times \int_{0}^{2 \pi} \exp \left(-\frac{\left(r \cos \theta-\hat{\mu}_{x}\right)^{2}}{2 \sigma_{x}^{2}}-\frac{\left(r \sin \theta-\hat{\mu}_{y}\right)^{2}}{2 \sigma_{y}^{2}}\right) d \theta .
\end{aligned}
$$

With the PDF of $r$, we can calculate the $n$th moment of $I_{p}$ as

$$
\mathbb{E}\left[I_{p}^{n}\right]=\mathbb{E}\left[A_{0}^{n} \exp \left(-\frac{2 n r^{2}}{w_{z_{e q}}^{2}}\right)\right]=A_{0}^{n} \mathcal{M}_{r^{2}}\left(-\frac{2 n}{w_{z_{e q}}^{2}}\right),
$$

where $\mathcal{M}_{r^{2}}($.$) is the moment-generating function (MGF) of$ the random variable $r^{2}$ and given by [34, Eq. (2.38)]

$$
\begin{aligned}
\mathcal{M}_{r^{2}}(\nu) & =\frac{1}{\sqrt{\left(1-\sigma_{x}^{2} \nu\right)\left(1-\sigma_{y}^{2} \nu\right)}} \\
& \times \exp \left(\frac{\hat{\mu}_{x}^{2} \nu}{1-2 \sigma_{x}^{2} \nu}+\frac{\hat{\mu}_{y}^{2} \nu}{1-2 \sigma_{y}^{2} \nu}\right) .
\end{aligned}
$$

Therefore, the $n$th moment of $I_{p}$ becomes in this case

$$
\begin{aligned}
\mathbb{E}\left[I_{p}^{n}\right] & =\frac{A_{0}^{n} \xi_{x} \xi_{y}}{\sqrt{\left(n+\xi_{x}^{2}\right)\left(n+\xi_{y}^{2}\right)}} \\
& \times f \exp \left(-\frac{2 n}{w_{z_{e q}}^{2}}\left[\frac{\hat{\mu}_{x}^{2}}{1+\frac{n}{\xi_{x}^{2}}}+\frac{\hat{\mu}_{y}^{2}}{1+\frac{n}{\xi_{y}^{2}}}\right]\right),
\end{aligned}
$$

where $\xi_{x}=\frac{w_{z e q}}{2 \sigma_{x}}$ and $\xi_{y}=\frac{w_{z e q}}{2 \sigma_{y}}$, are the ratio between the equivalent beam width and the jitter variance for each direction. From this relation, we can state that for high jitter variance, $\xi_{i}$ gets closer to zero and vice versa. Similarly, for wide beams, the effect of the pointing error is trivial and thus $\xi_{i} \rightarrow \infty$.

b) Rayleigh Distribution: When both displacement have zero mean and common variance as shown in Fig. $1 \mathrm{~b}$ (i.e. $\hat{\mu}_{x}=\hat{\mu}_{y}=0$ and $\left.\sigma_{x}=\sigma_{y}=\sigma\right), r$ is a Rayleigh distributed $\mathrm{RV}$ whose PDF is given by

$$
f_{r}(r)=\frac{r}{\sigma^{2}} \exp \left(-\frac{r^{2}}{2 \sigma^{2}}\right) .
$$

The PDF of $I_{p}$ reduces in this case to [14]

$$
f_{I_{p}}\left(I_{p}\right)=\frac{\xi^{2}}{A_{0}^{\xi^{2}}} I_{p}^{\xi^{2}-1}
$$

where $\xi=\frac{w_{z_{e q}}}{2 \sigma}$. The $n$th moment can be deduced from (20) as

$$
\mathbb{E}\left[I_{p}^{n}\right]=\frac{A_{0}^{n} \xi^{2}}{n+\xi^{2}} .
$$

c) Hoyt Distribution: Here, zero mean but different variances are assumed for the two displacements directions as shown in Fig. 1d (i.e. $\hat{\mu}_{x}=\hat{\mu}_{y}=0$ and $\sigma_{x} \neq \sigma_{y}$ ). In this case, $r$ becomes a Hoyt distributed RV with PDF given by

$$
f_{r}(r)=\frac{r}{q \sigma_{y}^{2}} \exp \left(-\frac{r^{2}\left(1+q^{2}\right)}{4 q^{2} \sigma_{y}^{2}}\right) I_{0}\left(\frac{r^{2}\left(1-q^{2}\right)}{4 q^{2} \sigma_{y}^{2}}\right),
$$

where $q=\frac{\sigma_{x}}{\sigma_{y}}=\frac{\xi_{y}}{\xi_{x}}$. This special case was studied in [15] and the PDF of $I_{p}$ was found to be given by

$$
\begin{aligned}
f_{I_{p}}\left(I_{p}\right) & =\frac{\xi_{x} \xi_{y}}{A_{0}}\left(\frac{I_{p}}{A_{0}}\right)^{\frac{\xi_{x}^{2}\left(1+q^{2}\right)}{2}-1} \\
& \times I_{0}\left(\frac{\xi_{x}^{2}\left(1-q^{2}\right)}{2} \ln \frac{I_{p}}{A_{0}}\right), \quad 0 \leq I_{p} \leq A_{0} .
\end{aligned}
$$

The $n$th moment can be deduced from (20) as

$$
\mathbb{E}\left[I_{p}^{n}\right]=\frac{A_{0}^{n} \xi_{x} \xi_{y}}{\sqrt{\left(\xi_{x}^{2}+n\right)\left(\xi_{y}^{2}+n\right)}} .
$$

d) Rician Distribution: If both displacements have distinct non-zero mean and common variance as shown in Fig. 1e (i.e. $\hat{\mu}_{x}+\hat{\mu}_{y} \neq 0$ and $\sigma_{x}=\sigma_{y}=\sigma$ ) then $r$ is a Rician distributed RV with PDF given by

$$
f_{r}(r)=\frac{r}{\sigma^{2}} \exp \left(\frac{-\left(r^{2}+s^{2}\right)}{2 \sigma^{2}}\right) I_{0}\left(\frac{r s}{\sigma^{2}}\right),
$$

where $s=\sqrt{\hat{\mu}_{x}^{2}+\hat{\mu}_{y}^{2}}$ and $I_{j}($.$) is the modified Bessel$ function of the first kind with order $j$. This case has been visited in [3] and the PDF of the pointing error has been derived as

$$
f_{I_{p}}\left(I_{p}\right)=\frac{\xi^{2} \exp \left(\frac{-s^{2}}{2 \sigma^{2}}\right)}{A_{0}^{\xi^{2}}} I_{p}^{\xi^{2}-1} I_{0}\left(\frac{s}{\sqrt{2} \sigma^{2}} \sqrt{w_{z_{e} q} \ln \left(\frac{A_{0}}{I_{p}}\right)}\right) .
$$


The $n$th moment can be deduced also from (20) as

$$
\mathbb{E}\left[I_{p}^{n}\right]=\frac{A_{0}^{n} \xi^{2}}{n+\xi^{2}} \exp \left(-\frac{2 n \xi^{2} s}{w_{z_{e q}}^{2}\left(n+\xi^{2}\right)}\right),
$$

which matches [3, Eq. 6].

e) Zero-mean Single-sided Gaussian Distribution: In this scenario, the displacement occur in only one direction either parallel or orthogonal to the detection plane as shown in Fig. 1c (i.e. $\hat{\mu}_{x}=\hat{\mu}_{y}=0, \sigma_{x}=\sigma$, and $\sigma_{y}=0$ ). This model was first introduced in [22] and the PDF of $I_{p}$ can be derived in this case by simple RV transformation of (16), yielding

$$
f_{I_{p}}\left(I_{p}\right)=\frac{\xi I_{p}^{\xi^{2}-1}}{A_{0}^{\xi^{2}} \sqrt{\pi \ln \left(\frac{A_{0}}{I_{p}}\right)}},
$$

and the resulting $n$th moment can be expressed as

$$
\mathbb{E}\left[I_{p}^{n}\right]=\frac{\xi A_{0}^{n}}{\sqrt{n+\xi^{2}}} .
$$

f) Non-zero Mean Single-sided Gaussian Distribution: In this case, assuming $\hat{\mu}_{x}=\hat{\mu}_{y}=\mu_{p}, \sigma_{x}=\sigma$, and $\sigma_{y}=0$ and we can obtain the PDF of $I_{p}$ as

$$
\begin{aligned}
f_{I_{p}}\left(I_{p}\right) & =I_{p}^{\xi^{2}-1} \xi^{2} \sqrt{\frac{\mu_{p} \sqrt{2}}{w_{z_{e q}} \sqrt{\ln \frac{A_{0}}{I_{p}}}}} \exp \left(-\frac{2 \mu_{p}^{2} \xi^{2}}{w_{z_{e q}}^{2}}\right) \\
& \times I_{-\frac{1}{2}}\left(\frac{2 \mu_{p} \xi^{2}}{w_{z_{e q}}} \sqrt{2 \ln \frac{A_{0}}{I_{p}}}\right),
\end{aligned}
$$

and then the $n$th moment can as a result be expressed as

$$
\mathbb{E}\left[I_{p}^{n}\right]=\frac{A_{0}^{n} \xi}{\sqrt{n+\xi^{2}}} \exp \left(-\frac{2 n \mu_{p}^{2}\left(n+2 \xi^{2}\right)}{w_{z_{e q}}^{2}\left(n+\xi^{2}\right)}\right) .
$$

\section{Performance Analysis: Asymptotic Ergodic CAPACITY}

Complete performance analysis of the FSO systems requires the statistics of the irradiance, which might not be mathematical tractable. For the purpose of this work, to study the asymptotic channel capacity of FSO links with general pointing error model, the $n$th moment of the irradiance is the only requirement.

At high SNR and considering that perfect channel-state information (CSI) is available at both the receiver and the transmitter of an FSO communication system, the ergodic capacity $\bar{C} \triangleq \underset{\gamma_{e n d}}{\mathbb{E}}\left[\log _{2}\left(1+\delta \gamma_{\text {end }}\right)\right]^{4}$ can be obtained through an asymptotic analysis by utilizing the moments of instantaneous end-to-end SNR, $\gamma_{\text {end }}$ [38, Eq. (8) and (9)] [18, Eq. (22)] as

$$
\left.\bar{C} \underset{\mu \gg 1}{\approx} \frac{\partial}{\partial n} \mathbb{E}\left[\gamma_{e n d}^{n}\right]\right|_{n=0} .
$$

\footnotetext{
${ }^{4}$ Although optical channel is well described as slow varying channel where coherence time is much greater than transmission time, ergodic capacity is still a widely adopted metric. The suitability of ergodic capacity as performance metric improves in this work as we consider pointing errors which typically increase the rate of channel fluctuations. In the case of heterodyne detection $(\mathrm{r}=1)$ the expression $\bar{C}=\mathbb{E}\left[\log _{2}\left(1+\delta \gamma_{\text {end }}\right)\right]$ represents the exact ergodic capacity where $\delta=1$. However, in the case of IM/DD $(r=2)$ the expression $\bar{C}=\mathbb{E}\left[\log _{2}(1+\delta \gamma)\right]$ where $\delta=e / 2 \pi$ represents a tight lower bound for capacity [35] [36, Eq. (26)] [37, Eq. (7.43)].
}

\section{A. Single Link Asymptotic Capacity:}

Assuming a single link FSO system. Since $I_{a}$ and $I_{p}$ are statistically independent processes, the moments of the irradiance and the electrical SNR can be written as

$$
\begin{aligned}
& \mathbb{E}\left[I^{n}\right]=\mathbb{E}\left[I_{a}^{n}\right] \mathbb{E}\left[I_{p}^{n}\right]=A_{0}^{n} \mathbb{E}\left[I_{a}^{n}\right] \mathcal{M}_{r^{2}}\left(-\frac{2 n}{w_{z_{e q}}^{2}}\right), \\
& \mathbb{E}\left[\gamma^{n}\right]=\left(\frac{\eta^{r}}{N_{0}}\right)^{n} \mathbb{E}\left[I^{r n}\right] .
\end{aligned}
$$

Taking the derivative of (36), the asymptotic capacity of a single link at high SNR can be written as

$$
\bar{C} \underset{\mu \gg 1}{\approx} \mathcal{W}-r \log \left(\mathbb{E}\left[I_{a}\right]\right)+\left.\frac{\partial}{\partial n} \mathbb{E}\left[I_{a}^{r n}\right]\right|_{n=0},
$$

such that

$$
\mathcal{W}=\log \left(\frac{\delta \mu A_{0}^{r}}{\mathbb{E}\left[I_{p}\right]^{r}}\right)-\frac{2 r}{w_{z_{e q}}} \mathcal{M}_{r^{2}}^{\prime}(0),
$$

where $\mathcal{M}_{r^{2}}($.$) is given in (19). Therefore, \mathcal{W}$ can take several forms as listed in Table II according to the specific pointing error model adopted. For example, for the most general case, $\mathcal{W}$ becomes

$$
\begin{aligned}
\mathcal{W} & =\log \left(\left[\frac{\left(1+\xi_{x}^{2}\right)\left(1+\xi_{y}^{2}\right)}{\xi_{x}^{2} \xi_{y}^{2}}\right]^{\frac{r}{2}} \delta \mu\right)-\frac{r}{2 \xi_{x}^{2}}-\frac{r}{2 \xi_{y}^{2}} \\
& -\frac{2 r}{w_{z_{e q}}^{2}}\left(\frac{\hat{\mu}_{x}^{2}}{1+\xi_{x}^{2}}+\frac{\hat{\mu}_{y}^{2}}{1+\xi_{y}^{2}}\right) .
\end{aligned}
$$

The result in Eq. (37) is generic that it can be applied to any turbulence channel model and only requires finding the moment of $I_{a}$ and its derivative. In this work, we consider weak and strong atmospheric turbulence modeled as LogNormal and Gamma-Gamma turbulences, respectively. Hence, the capacity of the channel can be asymptotically be found by taking the first derivatives of (10), (14) and substituting it in (37), we reach after some manipulations and simplifications the following results

$$
\begin{aligned}
& \left.\bar{C}\right|_{\Gamma \Gamma} \underset{\mu \gg 1}{\approx} \mathcal{W}-r \log (\alpha \beta)+r \psi(\alpha)+r \psi(\beta), \\
& \left.\bar{C}\right|_{\mathrm{LN}} \underset{\mu \gg 1}{\approx} \mathcal{W}-2 r \sigma_{X}^{2},
\end{aligned}
$$

for the Gamma-Gamma and the Log-Normal cases, respectively. This result can specialize to different cases according to the pointing error impairments model based on the choice of $\mathcal{W}$.

\section{B. Dual-Branch Diversity Link:}

In this section, we investigate the dual-aperture FSO system over correlated Log-Normal channels in which the footprint of the beam is shown in Fig. 2. The beam is originally pointing at $\mathcal{A}=\left[\begin{array}{ll}0 & 0\end{array}\right]^{T}$ while the apertures are placed in $\mathcal{B}_{1}=\left[\begin{array}{ll}\frac{d}{2} & 0\end{array}\right]^{T}$ and $\mathcal{B}_{2}=\left[\begin{array}{ll}-\frac{d}{2} & 0\end{array}\right]^{T}$ such that $d$ is the separation distance between the centers of the apertures. Note that due to building sway or other factors, the center of the beam moved to a random location $\mathcal{A}+\left[\begin{array}{ll}x & y\end{array}\right]^{T}$. Then, the distance to the center of the $i$ th aperture can be given as Eq. (15) (i.e. 
Table II: Different forms of $\mathcal{W}$ depends mainly on the pointing error model considered

\begin{tabular}{c||l}
\hline \hline Pointing Errors Model & $\mathcal{W}$ \\
\hline Beckmann $\left(\hat{\mu}_{x} \neq \hat{\mu}_{y}\right.$ and $\left.\xi_{x} \neq \xi_{y}\right)$ & $\log \left(\left[\frac{\left(1+\xi_{x}^{2}\right)\left(1+\xi_{y}^{2}\right)}{\xi_{x}^{2} \xi_{y}^{2}}\right]^{\frac{r}{2}} \delta \mu\right)-\frac{r}{2 \xi_{x}^{2}}-\frac{r}{2 \xi_{y}^{2}}-\frac{2 r}{w_{z e q}^{2}}\left(\frac{\hat{\mu}_{x}^{2}}{1+\xi_{x}^{2}}+\frac{\hat{\mu}_{y}^{2}}{1+\xi_{y}^{2}}\right)$ \\
\hline Rayleigh $\left(\hat{\mu}_{x}=\hat{\mu}_{y}=0\right.$ and $\left.\xi_{x}=\xi_{y}=\xi\right)$ & $\log \left(\left[\frac{1+\xi^{2}}{\xi^{2}}\right]^{r} \delta \mu\right)-\frac{r}{\xi^{2}}$ \\
\hline Rician $\left(\hat{\mu}_{x}+\hat{\mu}_{y} \neq 0\right.$ and $\left.\xi_{x}=\xi_{y}=\xi\right)$ & $\log \left(\left[\frac{1+\xi^{2}}{\xi^{2}}\right]^{r} \delta \mu\right)-\frac{r}{\xi^{2}}-\frac{2 r s^{2}}{w_{e q}^{2}}+\frac{2 r s^{2} \xi^{2}}{w_{z e q}^{2}\left(1+\xi^{2}\right)}$ \\
\hline Hoyt $\left(\hat{\mu}_{x}=\hat{\mu}_{y}=0\right.$ and $\left.\xi_{x} \neq \xi_{y}\right)$ & $\log \left(\left[\frac{1+\xi_{x}^{2}}{\xi_{x}^{2}}\right]^{\frac{r}{2}}\left[\frac{1+\xi_{y}^{2}}{\xi_{y}^{2}}\right]^{\frac{r}{2}} \delta \mu\right)-\frac{r}{2 \xi_{x}^{2}}-\frac{r}{2 \xi_{y}^{2}}$ \\
\hline Gaussian $\left(\hat{\mu}_{x}=0, \xi_{x}=\xi\right.$, and $\left.\xi_{y}=\infty\right)$ & $\log \left(\left[\frac{1+\xi^{2}}{\xi^{2}}\right]^{\frac{r}{2}} \delta \mu\right)-\frac{r}{2 \xi^{2}}$ \\
\hline Shifted Gaussian $\left(\hat{\mu}_{x}=\mu_{p}, \xi_{x}=\xi\right.$, and $\left.\xi_{y}=\infty\right)$ & $\log \left(\left[\frac{1+\xi^{2}}{\xi^{2}}\right]^{\frac{r}{2}} \delta \mu\right)-\frac{r}{2 \xi^{2}}-\frac{4 r \mu_{p}^{2}}{w_{z_{e q}}^{2}}+\frac{2 r \mu_{p}^{2}\left(1+2 \xi^{2}\right)}{w_{z_{e q}}^{2}\left(1+\xi^{2}\right)}$ \\
\hline No pointing errors & $\log (\delta \mu)$ \\
\hline
\end{tabular}

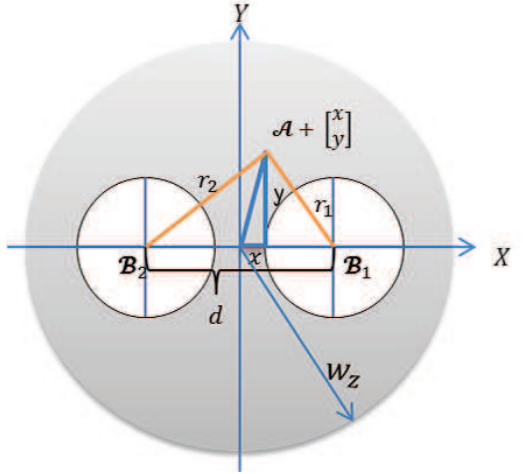

Figure 2: Beam foot print of the dual-aperture FSO system.

$r_{1}=\sqrt{\left(\frac{d}{2}-x\right)^{2}+y^{2}}$ and $\left.r_{2}=\sqrt{\left(\frac{d}{2}+x\right)^{2}+y^{2}}\right)$, which affect the pointing error. The received signals captured by the optical apertures will be combined together. More specifically, two combining techniques namely selection combining (SC) and switched and stay combining (SSC) are examined:

1) Selection-Combining (SC): In this technique, the branch with the larger instantaneous SNR is selected and therefore the output SNR can be written as

$$
\gamma_{S C}=\max \left(\gamma_{1}, \gamma_{2}\right)
$$

where $\gamma_{i}$ is defined in Eq. (3). Thus, Eq. (42) can be rewritten as

$$
\gamma_{S C}=\max \left(\frac{\eta I_{1}^{r} I_{p_{1}}^{r}}{N_{0}}, \frac{\eta I_{2}^{r} I_{p_{2}}^{r}}{N_{0}}\right)=\frac{\eta}{N_{0}} \max \left(I_{1} I_{p_{1}}, I_{2} I_{p_{2}}\right)^{r} .
$$

Note that $I_{1}$ and $I_{2}$ are two correlated Log-Normal variates with power correlation coefficient $\rho$ that is a function of the separation distance and the coherence length [39], [40], given by

$$
\rho=\exp \left[-\left(\frac{d}{\rho_{o}(z)}\right)^{\frac{-3}{5}}\right]
$$

where $\rho_{o}(z)=\left(1.46 C_{n}^{2} k^{2} z\right)^{(-3 / 5)}$ is the coherence length of a plane wave propagation ${ }^{5}$. It is clear from the expression that the links are correlated when the spacing between apertures is less than the coherence length of the beam.

The pointing error components $I_{p_{1}}$ and $I_{p_{2}}$ mainly depend on the distance $r_{i}$. Noting that $r_{2}^{2}=r_{1}^{2}+2 d x$, we can rewrite Eq. (43) as

$$
\begin{aligned}
& \gamma_{S C}=\frac{\eta}{N_{0}} \max \left(I_{1} A_{0} \exp \left(\frac{-2 r_{1}^{2}}{w_{z_{e q}}^{2}}\right), I_{2} A_{0} \exp \left(\frac{-2 r_{2}^{2}}{w_{z_{e q}}^{2}}\right)\right)^{r} \\
& =\frac{\eta}{N_{0}} \max \left(I_{1} A_{0} \exp \left(\frac{-2 r_{1}^{2}}{w_{z_{e q}}^{2}}\right), I_{2} A_{0} \exp \left(\frac{-2\left(r_{1}^{2}+2 d x\right)}{w_{z_{e q}}^{2}}\right)\right)^{r} \\
& =\frac{\eta}{N_{0}}\left(A_{0} \exp \left(\frac{-2 r_{1}^{2}}{w_{z_{e q}}^{2}}\right)\right)^{r} \max \left(I_{1}, I_{2} \exp \left(\frac{-4 d x}{w_{z_{e q}}^{2}}\right)\right)^{r} \\
& =\frac{\eta}{N_{0}} I_{p_{1}} \max \left(I_{1}, \hat{I}_{2}\right)^{r}=\frac{\eta}{N_{0}} I_{p_{1}}^{r} I_{S C}^{r},
\end{aligned}
$$

where $\hat{I}_{2}=\exp \left(2 X_{2}-\frac{4 d}{w_{z_{e q}}^{2}} x\right)$ leading to $\hat{I}_{2} \sim$ $\ln \mathcal{N}\left(-2 \sigma_{x_{2}}^{2}-\lambda_{1}, 4 \sigma_{x_{2}}^{2}+\lambda_{2}^{2}\right)$ such that $\lambda_{1}=\frac{4 d \mu_{x}}{w_{z_{e q}}^{2}}$ and $\lambda_{2}=\frac{4 d \sigma_{x}}{w_{z e q}^{2}}$. Furthermore, the $n$th moment of $\gamma_{S C}$ can be expressed as

$$
\mathbb{E}\left[\gamma_{S C}^{n}\right]=\frac{\mathbb{E}\left[I_{p_{1}}^{r n}\right] \mathbb{E}\left[I_{S C}^{r n}\right]}{\mathbb{E}\left[I_{p_{1}}\right]^{r n} \mathbb{E}\left[I_{S C}\right]^{r n}} \mu_{S C}^{n},
$$

where $\mathbb{E}\left[I_{p_{1}}^{n}\right]$ can be derived using Eq. (20), and $\mathbb{E}\left[I_{S C}^{n}\right]$ can be expressed shown in Eq. (47), where $\zeta=\lambda_{1}-2 \sigma_{x_{1}}^{2}+2 \sigma_{x_{2}}^{2}$, $\kappa=\lambda_{2}^{2}+4 \sigma_{x_{2}}^{2}, \phi=\frac{2 \sigma_{x_{1}}}{\sqrt{\kappa}}$, and $\mathcal{P}_{1}^{2}=f\left(2 \sigma_{x_{1}}, \sqrt{\kappa},-\rho\right)$ such that $f(a, b, c)=a^{2}+b^{2}+2 a b c$.

Based on Eq. (37), the asymptotic capacity of dual branch FSO system with SC can be written as shown in Eq. (48)

where $\mathcal{P}_{2}=f\left(\sigma_{x_{1}}, \sigma_{x_{2}},-\rho\right)$.

2) Switched and Stay Combining (SSC): The SNR $\gamma_{S S C}$ at the output of a dual-branch SSC receiver is given by [34],

\footnotetext{
${ }^{5}$ Physically, the coherence parameter is the measure of light coherence across each transverse plane along the propagation path [41]. The coherence length of a spherical wave propagation is equal to $\rho_{o}(z)=$ $\left(0.55 C_{n}^{2} k^{2} z\right)^{(-3 / 5)}$ [7, Eq. (65)].
} 


$$
\mathbb{E}\left[I_{S C}^{n}\right]=\exp \left(\frac{1}{2} n^{2} \kappa-n\left(\lambda_{1}+2 \sigma_{x_{2}}^{2}\right)\right) Q\left(\frac{\zeta-n \kappa(1-\rho \phi)}{\mathcal{P}_{1}}\right)+\exp \left(2 n^{2} \sigma_{x_{1}}^{2}-2 n \sigma_{x_{1}}^{2}\right) Q\left(\frac{-\zeta-4 n \sigma_{x_{1}}^{2}\left(1-\rho \phi^{-1}\right)}{\mathcal{P}_{1}}\right)
$$

$$
\begin{gathered}
\left.\bar{C}\right|_{S C} \underset{\mu \gg 1}{\approx} \mathcal{W}+\frac{r}{\sqrt{2 \pi}} \exp \left(\frac{-\zeta^{2}}{2 \mathcal{P}_{1}^{2}}\right) \sqrt{\lambda_{2}^{2}+4 \lambda_{2}\left(\sigma_{x_{2}}-\rho \sigma_{x_{1}}\right)+4 \mathcal{P}_{2}}-2 r \sigma_{x_{1}}^{2}-r \zeta Q\left(\frac{\zeta}{\mathcal{P}_{1}}\right) \\
-r \log \left[Q\left(\frac{-\zeta-4 \sigma_{x_{1}}^{2}\left(1-\rho \phi^{-1}\right)}{\mathcal{P}_{1}}\right)+\exp \left(-\left(\zeta+2 \sigma_{x_{1}}^{2}\right)+\frac{1}{2} \kappa^{2}\right) Q\left(\frac{\zeta-\kappa(1-\rho \phi)}{\mathcal{P}_{1}}\right)\right] .
\end{gathered}
$$

[42]

$$
\gamma_{S S C}=\left\{\begin{array}{ll}
\gamma_{1}, & \gamma_{1} \geq \gamma_{t} \\
\gamma_{2}, & \gamma_{1}<\gamma_{t}
\end{array}= \begin{cases}\frac{\eta I_{1}^{r} I_{p_{1}}^{r}}{N} & I_{1} I_{p_{1}} \geq I_{t} \\
\frac{\eta I_{2}^{r} I_{p_{2}}^{r}}{N_{0}} & I_{1} I_{p_{1}}<I_{t}\end{cases}\right.
$$

where $\gamma_{t}$ is the switching threshold below which the receiver switches to the other diversity branch and $I_{t}=\sqrt[r]{\frac{N_{0} \gamma_{t}}{\eta}}$. Following similar analysis in Sec. III-B1, we have $\gamma_{S S C}=$ $\frac{\eta}{N_{0}} I_{p_{1}}^{r} I_{S S C}^{r}$, where $I_{S S C}^{r}$ is given by .

$$
I_{S S C}= \begin{cases}I_{1}, & I_{p_{1}} I_{1} \geq I_{t} \\ \hat{I}_{2}, & I_{p_{1}} I_{1}<I_{t}\end{cases}
$$

Then the $n$th moment of the $\gamma_{S S C}$ can be expressed as

$$
\mathbb{E}\left[\gamma_{S S C}^{n}\right]=\frac{\mathbb{E}\left[I_{p_{1}}^{r n}\right] \mathbb{E}\left[I_{S S C}^{n}\right]}{\mathbb{E}\left[I_{p_{1}}\right]^{r n} \mathbb{E}\left[I_{S S C}\right]^{n}} \mu^{n},
$$

where the moments of $I_{S S C}$ is given as in Eq. (56) utilizing earlier results in [43] such that

$$
\begin{aligned}
\mathcal{K}_{1} & =\frac{2 \sigma_{x_{1}}^{2}+\log \left(\gamma_{t}\right)}{2 \sigma_{x_{1}}}, \\
\mathcal{K}_{2} & =\lambda_{1}+2 \sigma_{x_{2}}^{2}+\log \left(\gamma_{t}\right), \\
\varepsilon_{1} & =\frac{Q\left(-\mathcal{K}_{1}\right)}{Q\left(-\mathcal{K}_{1}\right)+Q\left(-\frac{\mathcal{K}_{2}}{\sqrt{\kappa}}\right)}, \\
\varepsilon_{2} & =\frac{Q\left(-\frac{\mathcal{K}_{2}}{\sqrt{\kappa}}\right)}{Q\left(-\mathcal{K}_{1}\right)+Q\left(-\frac{\mathcal{K}_{2}}{\sqrt{\kappa}}\right)},
\end{aligned}
$$

Since the moment of the irradiance $I_{S S C}$ is available, we can directly apply Eq. (37) to get the asymptotic capacity for SSC case as in Eq. (57).

\section{Numerical Simulations}

In this section, we validate and evaluate our analytical expressions of the channel capacity for two systems, single link FSO and dual-aperture FSO system over correlated LogNormal channels. It is important to mention that Monte-Carlo computer based simulations are utilized to obtain all exact results. In each plot, we specify all parameters considered in the simulation i.e. the beamwaist, $w_{0}$, phase front $F_{0}$, the distance $z$, aperture radius $a$, refractive index $C_{n}^{2}$, jitter variances $\sigma_{x}^{2}$ $\sigma_{y}^{2}$ and boresight mean $\mu_{x}$ and $\mu_{y}$. First, we compare between the single link and dual-aperture FSO systems for different

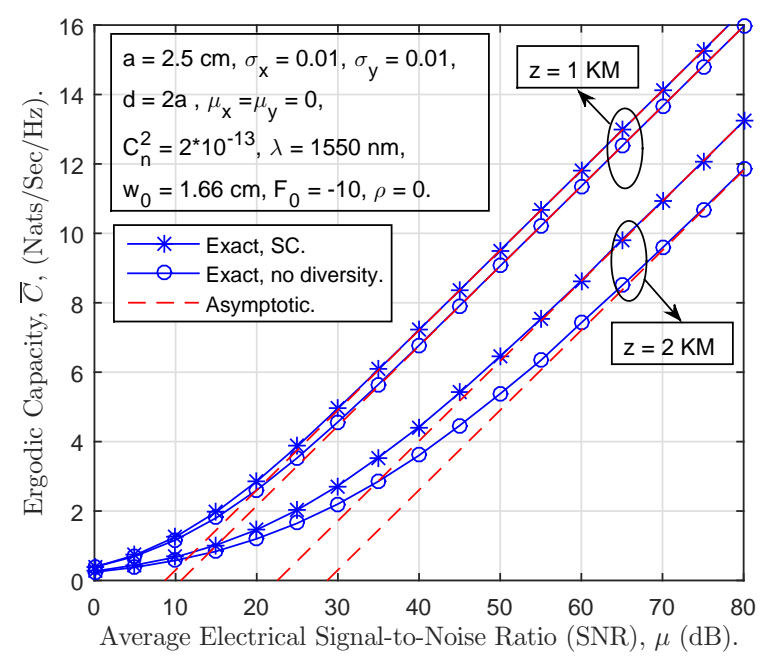

Figure 3: Comparison between the channel capacity of a single aperture and dual-aperture FSO systems.

distances, $z$, as shown in Fig. 3. It is expected that the diversity link can enhance the capacity when the turbulence conditions get severe since the distance is directly proportional to the Rytov variance (i.e. as distance increases, more turbulence eddies are added). Also, our asymptotic results are tight at high SNR. However, as the channel gets more turbulent, the convergence of the asymptotic results to the exact ones happen at larger SNR. In Fig. 4 we plot the ergodic capacity of a single link versus the average electrical SNR over weak turbulence modeled as Log-normal fading channel. Assuming no-boresight, we consider different scenarios of jitter variance i.e. high pointing error effect is presented by high values of $\sigma_{x}^{2}$ and $\sigma_{y}^{2}$. Clearly, highly varying beam due to winds or other factor results in performance degradation. Interestingly, our asymptotic analytical results show an excellent match with the exact ones generated by Monte-Carlo computer based simulation. Next, we show the effect of beam waist at the transmitter on the capacity of the link in Fig. 5. First thing to observe is that our asymptotic results converge well to the ones generated by Monte-Carlo simulation. Wide beam delivers the best result as it resists the pointing errors in contrast with the narrow beam. Lastly for the single link, we compare between unidirectional and bidirectional misalignments over Gamma- 


$$
\begin{aligned}
\mathbb{E}\left(I_{S S C}^{n}\right) & =\varepsilon_{1} \exp \left(2 n^{2} \sigma_{x_{1}}^{2}-2 n \sigma_{x_{1}}^{2}\right)\left(Q\left(2 n \rho \sigma_{x_{1}}-\mathcal{K}_{1}\right)+Q\left(\mathcal{K}_{1}-2 n \sigma_{x_{1}}\right)\right) \\
& +\gamma_{t}^{n} \varepsilon_{2} \exp \left(\frac{\kappa n^{2}}{2}-n \mathcal{K}_{2}\right)\left(Q\left(\frac{\kappa n \rho-\mathcal{K}_{2}}{\sqrt{\kappa}}\right)+Q\left(\frac{-\kappa n+\mathcal{K}_{2}}{\sqrt{\kappa}}\right)\right) .
\end{aligned}
$$

$$
\begin{aligned}
& \left.\bar{C}\right|_{\mathrm{SSC}} \underset{\mu \gg 1}{\approx} \mathcal{W}+\frac{r(1-\rho)}{\sqrt{\pi}}\left(\sqrt{2} \varepsilon_{1} \sigma_{x_{1}} \exp \left(\frac{-\mathcal{K}_{1}^{2}}{2}\right)+\frac{\varepsilon_{2} \sqrt{\kappa}}{\sqrt{2 \pi}} \exp \left(\frac{-\mathcal{K}_{2}^{2}}{2 \kappa}\right)\right)-r\left(\varepsilon_{1} \sigma_{x_{1}}^{2}+\varepsilon_{2} \sigma_{x_{2}}^{2}+\frac{1}{2} \varepsilon_{2} \lambda_{1}\right) \\
& -r \log \left[\varepsilon_{1} Q\left(-2 \sigma_{x_{1}}+\sigma_{x_{1}} \mathcal{K}_{1}\right)+\varepsilon_{1} Q\left(\frac{2 \rho \sigma_{x_{1}}^{2}-\mathcal{K}_{1}}{\sigma_{x_{1}}}\right)+\varepsilon_{2} \exp \left(\frac{\lambda_{2}^{2}-2 \lambda_{1}}{2}\right)\left(Q\left(\frac{\rho \kappa-\mathcal{K}_{2}}{\sqrt{\kappa}}\right)+Q\left(\frac{-\kappa+\mathcal{K}_{2}}{\sqrt{\kappa}}\right)\right)\right] .
\end{aligned}
$$

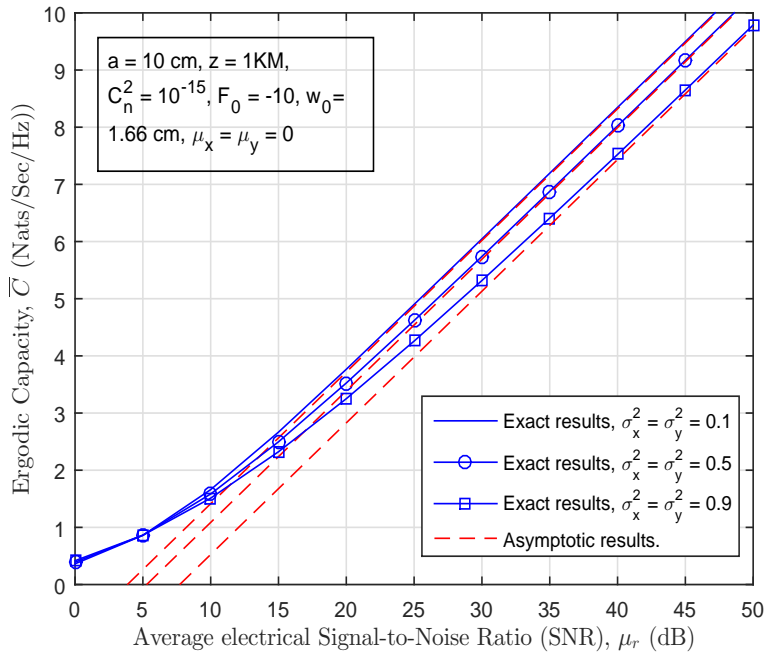

Figure 4: The effect of jitter variance on the capacity of a single link over Log-Normal turbulence.

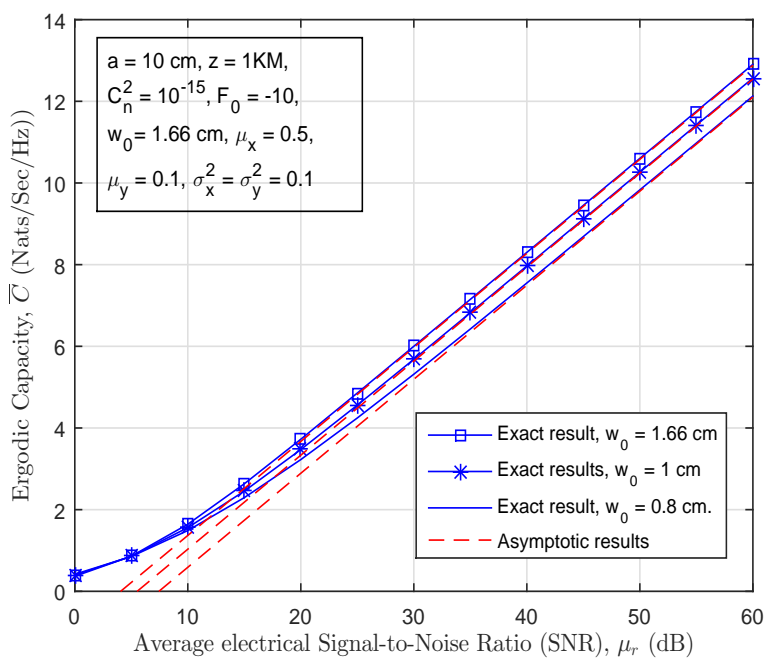

Figure 5: The effect of beam waist at the transmitter on the capacity over Log-Normal turbulence under boresight error.

Gamma turbulence in Fig. 6. Clearly, for low jitter variance i.e. high values of $\xi$, both directions of misalignment have

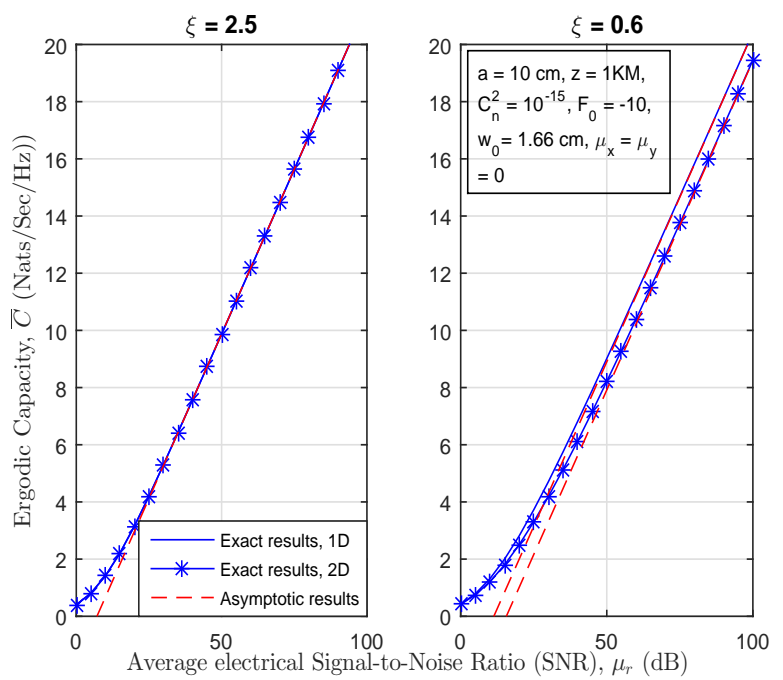

Figure 6: Comparison between unidirectional and bidirectional misalignment over Gamma-Gamma turbulence.

the same effect of the channel capacity. In contrast with high jitter variance case, channel capacity is less affected by the unidirectional misalignment.

With regard to diversity link, first we show the effect of boresight on the channel capacity of selection combined FSO link with two scenarios of jitter variance and beam waist in Fig. 7 and Fig. 8, respectively. We can conclude that low varying beam and wide beam are more resistant to the boresight.

The main purpose of diversity is to overcome scintillation. It can also help suppressing the effect of beam boresight error. From our observation in Fig. 9, with small boresight error, the distance does not make any difference to the channel capacity. On the other hand, larger boresight, spacing distance can be helpful in diminishing the effect of boresight. Finally, we show in Fig. 10 the effect of the pointing error on the channel capacity if SSC are employed to combine the signal at the receive side. Here, we consider only unidirectional misalignment, so we can obtain the optimal threshold $\gamma_{t}=\exp \left(-2 \sigma_{X}^{2}\right)$. It is clear that even with the misalignment being in one direction, performance degradation is expected. 


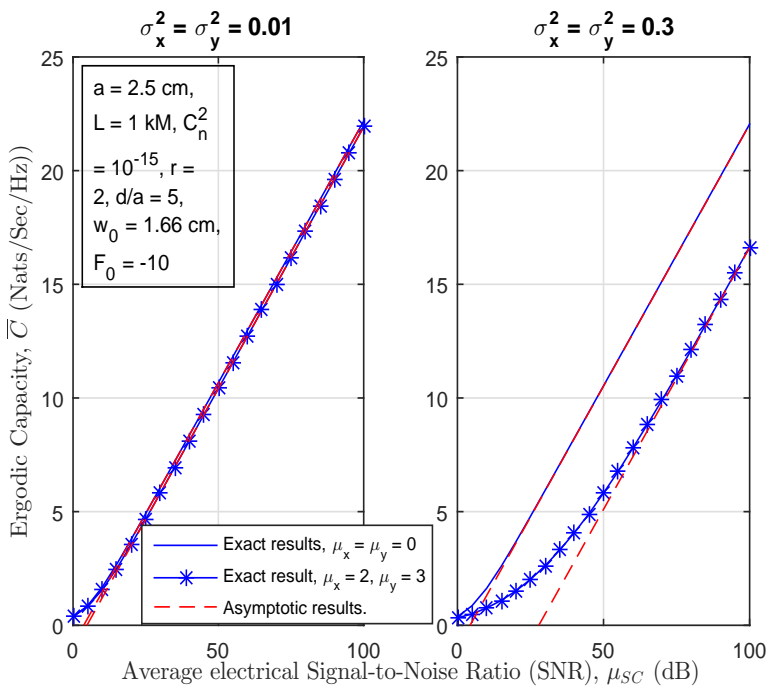

Figure 7: The effect of boresight and jitter variance on the channel capacity of dual-branch FSO link.

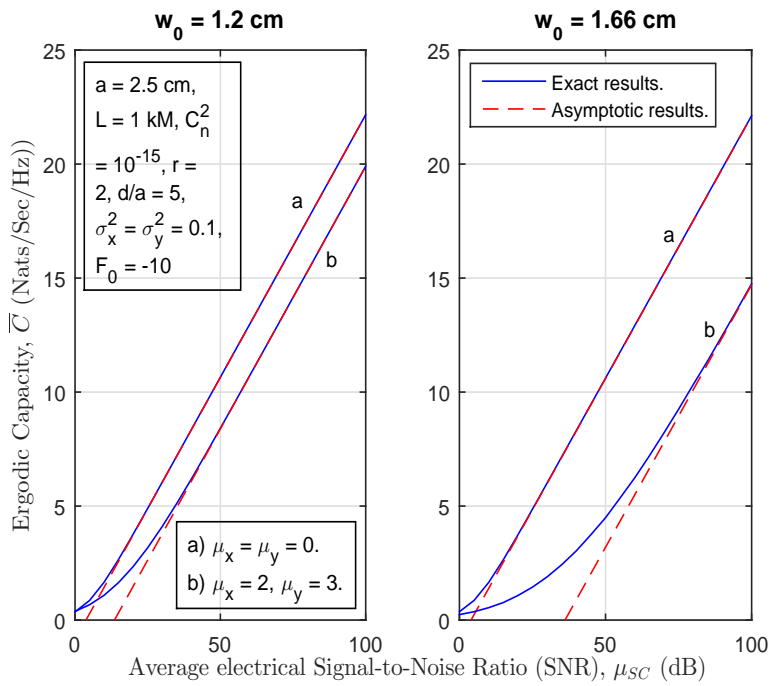

Figure 8: The effect of boresight and beam waist on the channel capacity of dual-branch FSO link.

\section{CONCLUSION}

In this work, we developed a general model for the pointing errors affecting FSO systems based on the generalized Beckmann distribution. Our result is generic and includes previously published models as special cases. Furthermore, due to the interest in the high SNR regime, we derived a general expression for the asymptotic ergodic capacity of FSO systems subject to generalized pointing error impairments. In this work, we have provided closed-form expressions of the Log-Normal and Gamma-Gamma channels. However, our formula can be applied to any turbulence channel such as Kdistributed, double Weibull, double Generalized Gamma and $\mathcal{M}$ channels. Actually our approach can be extended as well to diversity links. In particular, we have derived the asymptotic capacity of dual-aperture FSO system over correlated LogNormal channels. Numerical results, validated by computer simulations, show that our asymptotic results can accurately

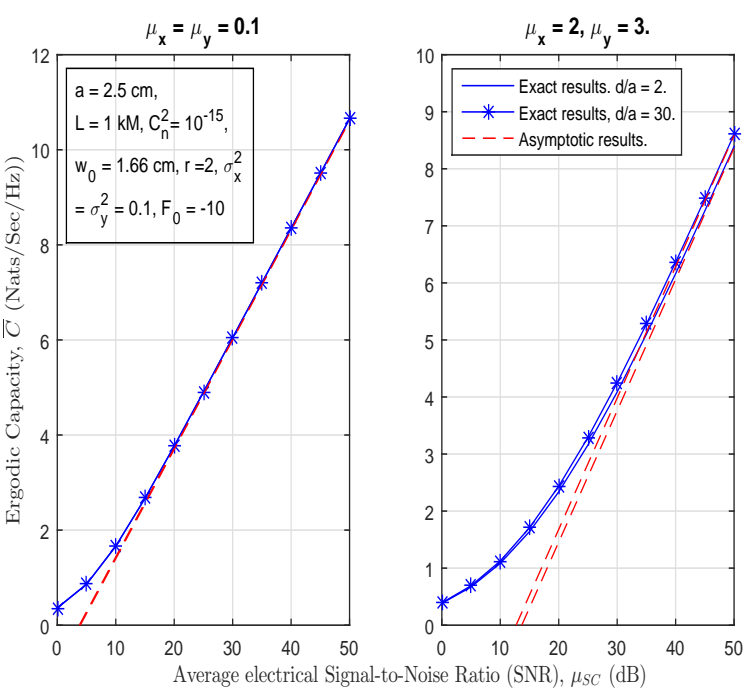

Figure 9: The effect of distance and boresight on the channel capacity of dual-branch FSO link.

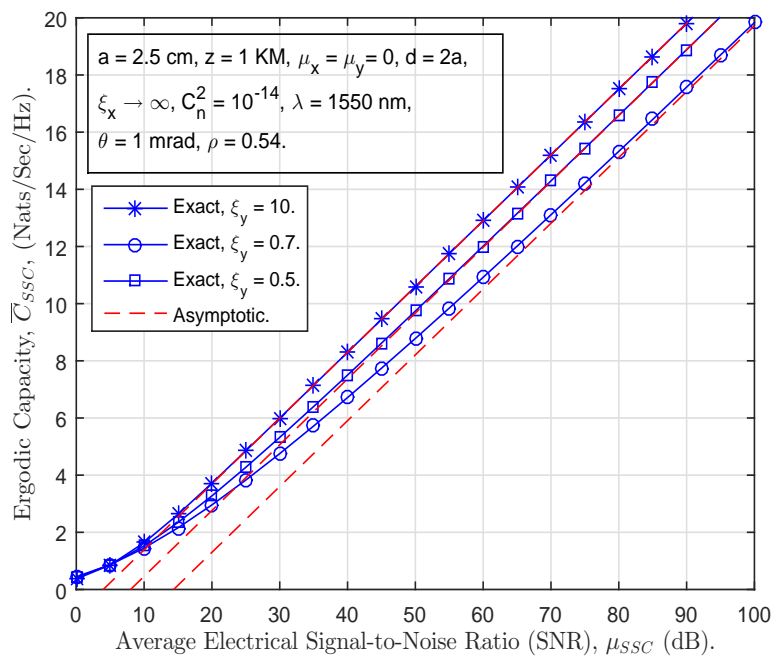

Figure 10: Unidirectional misalignment and corresponding channel capacity for the dual-aperture FSO system.

predict the performance of FSO systems in the high SNR regime.

\section{REFERENCES}

[1] S. Karp, R. Gagliardi, S. Moran, and L. Stotts, Optical Channels: Fibers, Clouds, Water, and the Atmosphere, ser. Applications of Communications Theory. Springer US, 1988.

[2] D. K. Borah and D. G. Voelz, "Pointing error effects on free-space optical communication links in the presence of atmospheric turbulence," Journal of Lightwave Technology, vol. 27, no. 18, pp. 3965-3973, Sep. 2009.

[3] F. Yang, J. Cheng, and T. Tsiftsis, "Free-space optical communication with nonzero boresight pointing errors," IEEE Transactions on Communications, vol. 62, no. 2, pp. 713-725, Feb. 2014.

[4] E. Lee and V. Chan, "Part 1: Optical communication over the clear turbulent atmospheric channel using diversity," IEEE Journal on Selected Areas in Communications, vol. 22, no. 9, pp. 1896-1906, Nov. 2004.

[5] T. Tsiftsis, H. Sandalidis, G. Karagiannidis, and M. Uysal, "Optical wireless links with spatial diversity over strong atmospheric turbulence channels," IEEE Transactions on Communications, vol. 8, no. 2, pp. 951-957, Feb. 2009. 
[6] G. Yang, M. A. Khalighi, S. Bourennane, and Z. Ghassemlooy, "Fading correlation and analytical performance evaluation of the space-diversity free-space optical communications system," Journal of Optics, vol. 16, no. 3, p. 035403 , Feb. 2014.

[7] L. C. Andrews, R. L. Phillips, and C. Y. Hopen, Laser Beam Scintillation with Applications. SPIE press, 2001, vol. 99 .

[8] M. Al-Habash, L. C. Andrews, and R. L. Phillips, "Mathematical model for the irradiance probability density function of a laser beam propagating through turbulent media," Optical Engineering, vol. 40, no. 8, pp. 1554-1562, Feb. 2001.

[9] X. Zhu and J. Kahn, "Free-space optical communication through atmospheric turbulence channels," IEEE Transactions on Communications, vol. 50, no. 8, pp. 1293-1300, Aug. 2002.

[10] T. Tsiftsis, "Performance of heterodyne wireless optical communication systems over Gamma-Gamma atmospheric turbulence channels," Electronics Letters, vol. 44, no. 5, pp. 372-373, Feb. 2008.

[11] H. Nistazakis, T. Tsiftsis, and G. Tombras, "Performance analysis of free-space optical communication systems over atmospheric turbulence channels," IET Communications, vol. 3, no. 8, pp. 1402-1409, Aug. 2009.

[12] A. Prokeš, "Modeling of atmospheric turbulence effect on terrestrial FSO link," Radio Eng, vol. 18, no. 1, pp. 42-47, Jan. 2009.

[13] X. Tang, S. Rajbhandari, W. Popoola, Z. Ghassemlooy, E. Leitgeb, S. Muhammad, and G. Kandus, "Performance of BPSK subcarrier intensity modulation free-space optical communications using a Log-Normal atmospheric turbulence model," in 2010 Symposium on Photonics and Optoelectronic (SOPO), Chengdu, China, June 2010, pp. 1-4.

[14] A. Farid and S. Hranilovic, "Outage capacity optimization for free-space optical links with pointing errors," IEEE/OSA Journal of Lightwave Technology, vol. 25, no. 7, pp. 1702-1710, July 2007.

[15] W. Gappmair, S. Hranilovic, and E. Leitgeb, "OOK performance for terrestrial FSO links in turbulent atmosphere with pointing errors modeled by Hoyt distributions," IEEE Communications Letters, vol. 15, no. 8, pp. 875-877, July 2011 .

[16] P. Beckmann, "Statistical distribution of the amplitude and phase of a multiply scattered field," Journal of research of the National Bureau of Standards-D.Radio propagation, vol. 66D, no. 3, June 1962.

[17] H. AlQuwaiee, H.-C. Yang, and M.-S. Alouini, "On the asymptotic ergodic capacity of FSO links with generalized pointing error model," in 2015 IEEE International Conference on Communications, (ICC 2015), London, UK, June 2015, pp. 5072-5077.

[18] I. S. Ansari, F. Yilmaz, and M.-S. Alouini, "A unified performance of free-space optical links over Gamma-Gamma turbulence channels with pointing errors," in Proceedings of IEEE 81st Vehicular Technology Conference (VTC Spring' 2015), Glasgow, Scotland, May 2015.

[19] H. AlQuwaiee, I. Ansari, and M.-S. Alouini, "On the performance of free-space optical communication systems over double generalized Gamma channel," IEEE Journal on Selected Areas in Communications, vol. 33, no. 9, pp. 1829-1840, May. 2015.

[20] I. Ansari, F. Yilmaz, and M.-S. Alouini, "Performance analysis of freespace optical links over Malaga $(\mathcal{M})$ turbulence channels with pointing errors," IEEE Transactions on Wireless Communications, vol. PP, no. 99 pp. 1-1, Aug. 2015.

[21] S. M. Navidpour, M. Uysal, and M. Kavehrad, "BER performance of free-space optical transmission with spatial diversity," IEEE Transactions on Wireless Communications, vol. 6, no. 8, pp. 2813-2819, Aug. 2007.

[22] A. Farid and S. Hranilovic, "Diversity gain and outage probability for MIMO free-space optical links with misalignment," IEEE Transactions on Communications, vol. 60, no. 2, pp. 479 - 487, Feb. 2012.

[23] H. Moradi, H. Refai, and P. LoPresti, "Switch-and-stay and switchand-examine dual diversity for high-speed free-space optics links," IET Optoelectronics, vol. 6, no. 1, pp. 34-42, Feb. 2012.

[24] M. Abaza, R. Mesleh, A. Mansour, and E.-H. M. Aggoune, "Diversity techniques for a free-space optical communication system in correlated Log-Normal channels," Optical Engineering, vol. 53, no. 1, pp. 016102 016 102, Jan. 2014

[25] H. E. Nistazakis, E. A. Karagianni, A. D. Tsigopoulos, M. E. Fafalios, and G. S. Tombras, "Average capacity of optical wireless communication systems over atmospheric turbulence channels," Journal of Lightwave Technology, vol. 27, no. 8, pp. 974-979, April 2009.

[26] H. Sandalidis and T. Tsiftsis, "Outage probability and ergodic capacity of free-space optical links over strong turbulence," Electronics Letters, vol. 44, no. 1, pp. 46-47, Jan. 2008.

[27] W. Gappmair, "Further results on the capacity of free-space optical channels in turbulent atmosphere," IET Communications, vol. 5, no. 9 , pp. 1262-1267, June 2011
[28] K. P. Peppas, A. N. Stassinakis, G. K. Topalis, H. E. Nistazakis, and G. S. Tombras, "Average capacity of optical wireless communication systems over IK atmospheric turbulence channels," Journal of Optical Communications and Networking, vol. 4, no. 12, pp. 1026-1032, Dec. 2012.

[29] "Performance estimation of free space optical links over negative exponential atmospheric turbulence channels," Optik - International Journal for Light and Electron Optics, vol. 122, no. 24, pp. 2191 - 2194, Dec. 2011.

[30] C. Liu, Y. Yao, Y. Sun, and X. Zhao, "Average capacity for heterodyne FSO communication systems over gamma-gamma turbulence channels with pointing errors," Electronics letters, vol. 46, no. 12, pp. 851-853, June 2010.

[31] N. Perlot, Characterization of Signal Fluctuations in Optical Communications with Intensity Modulation and Direct Detection Through the Turbulent Atmospheric Channel, ser. Berichte aus der Kommunikationstechnik. Shaker, 2006.

[32] I. Wolfram, Mathematica Edition: Version 8.0. Champaign, Illinois: Wolfram Research Inc., 2010.

[33] L. C. Andrews and R. L. Phillips, Laser Beam Propagation through Random Media. SPIE press, 2005, vol. 152

[34] M. K. Simon and M.-S. Alouini, Digital Communication over Fading Channels. New York: Wiley, 2000.

[35] A. Chaaban, J. M. Morvan, and M. S. Alouini, "Free-space optical communications: Capacity bounds, approximations, and a new spherepacking perspective," IEEE Transactions on Communications, vol. 64, no. 3, pp. 1176-1191, March 2016.

[36] A. Lapidoth, S. Moser, and M. Wigger, "On the capacity of free-space optical intensity channels," IEEE Transactions on Information Theory, vol. 55, no. 10, pp. 4449-4461, Oct. 2009.

[37] S. Arnon, J. Barry, G. Karagiannidis, R. Schober, and M. Uysal, Advanced Optical Wireless Communication Systems. Cambridge University Press, 2012.

[38] F. Yilmaz and M.-S. Alouini, "Novel asymptotic results on the highorder statistics of the channel capacity over generalized fading channels," in Proceedings of IEEE 13th International Workshop on Signal Processing Advances in Wireless Communications (SPAWC' 2012), Cesme, Turkey, June 2012, pp. 389-393.

[39] X. Zhu and J. M. Kahn, "Maximum-likelihood spatial-diversity reception on correlated turbulent free-space optical channels," in IEEE Global Telecommunications Conference (GLOBECOM'O0), vol. 2, San Francisco, CA, Dec. 2000, pp. 1237-1241.

[40] G. R. Osche, "Optical detection theory for laser applications," Optical Detection Theory for Laser Applications, by Gregory R. Osche, pp. 424. ISBN 0-471-22411-1. Wiley-VCH, July 2002., vol. 1, 2002.

[41] J. C. Ricklin and F. M. Davidson, "Atmospheric turbulence effects on a partially coherent Gaussian beam: Implications for free-space laser communication," J. Opt. Soc. Am. A, vol. 19, no. 9, pp. 1794-1802, Sep. 2002

[42] M. Blanco and K. Zdunek, "Performance and optimization of switched diversity systems for the detection of signals with rayleigh fading," IEEE Transactions on Communications, vol. 27, no. 12, pp. 1887-1895, Dec. 1979.

[43] M.-S. Alouini and M. K. Simon, "Dual diversity over correlated LogNormal fading channels," IEEE Transactions on Wireless Communications, vol. 50, no. 12, pp. 1946-1959, Dec. 2002.

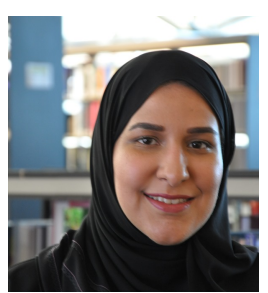

Hessa AlQuwaiee (S'09) received the B.S degree in Computer Engineering from Prince Mohamed University (PMU), Alkhobar, Saudi Arabia in 2011. In 2008, she was awarded the discovery scholarship to complete graduate studies in King Abdullah University of Science and Technology (KAUST). In 2013, she earned her M.S. degree in Electrical Engineering. She is now toward her PhD degree at KAUST. Her current research interests include, but not limited to, channel characterization and performance analysis of optical wireless communications, body-to-body communications, diversity combining techniques and MIMO systems. 


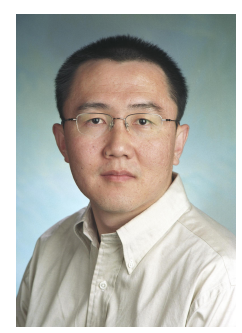

Hong-Chuan Yang (S'00, M'03, SM'07) received his $\mathrm{Ph} . \mathrm{D}$. degree in Electrical Engineering from the University of Minnesota, Minneapolis, USA, in 2003. Since September 2003, Dr. Yang has been with the Department of Electrical and Computer Engineering at the University of Victoria, Victoria, B.C., Canada, where he is now a professor. From 1995 to 1998 , Dr. Yang was a Research Associate at the Science and Technology Information Center (STIC) of Ministry of Posts \& Telecomm. (MPT), Beijing, China. His research interest include wireless channel modeling, diversity techniques, system performance evaluation, crosslayer design and energy efficient communications. He is an Editor for IEEE Transactions on Communications

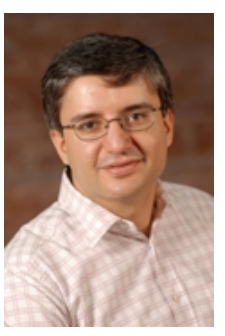

Mohamed-Slim Alouini (S'94, M'98, SM'03, F'09) was born in Tunis, Tunisia. He received the Ph.D. degree in Electrical Engineering from the California Institute of Technology (Caltech), Pasadena, CA, USA, in 1998. He served as a faculty member in the University of Minnesota, Minneapolis, MN, USA, then in the Texas A\&M University at Qatar, Education City, Doha, Qatar before joining King Abdullah University of Science and Technology (KAUST), Thuwal, Makkah Province, Saudi Arabia as a Professor of Electrical Engineering in 2009. His current research interests include the modeling, design, and performance analysis of wireless communication systems. 